\title{
Preservation of fertility in patients with cancer (Review)
}

\author{
SOFÍA DEL-POZO-LÉRIDA ${ }^{1}$, CRISTINA SALVADOR ${ }^{2}$, FINA MARTÍNEZ-SOLER ${ }^{3}$, AVELINA \\ TORTOSA $^{3}$, MANUEL PERUCHO ${ }^{4,5}$ and PEPITA GIMÉNEZ-BONAFÉ ${ }^{1}$
}

\footnotetext{
${ }^{1}$ Department of Physiological Sciences, Physiology Unit, Faculty of Medicine and Health Sciences, Bellvitge Campus, Universitat de Barcelona, IDIBELL, L'Hospitalet del Llobregat, 08907 Barcelona; ${ }^{2}$ Department of Gynecology, Gynecological Endocrinology and Reproduction Unit, Hospital Universitari Sant Joan de Déu, Esplugues de Llobregat, 08950 Barcelona; ${ }^{3}$ Department of Basic Nursing, Faculty of Medicine and Health Sciences, Universitat de Barcelona, IDIBELL, L' Hospital et del Llobregat, 08907 Barcelona, Spain; ${ }^{4}$ Sanford Burnham Prebys Medical Discovery Institute (SBP), La Jolla, CA 92037, USA; ${ }^{5}$ Program of Predictive and Personalized Medicine of Cancer (PMPPC), of the Research Institute Germans Trias i Pujol (IGTP), Badalona, 08916 Barcelona, Spain
}

Received January 18, 2019; Accepted March 6, 2019

DOI: 10.3892/or.2019.7063

\begin{abstract}
Survival rates in oncological patients have been steadily increasing in recent years due to the greater effectiveness of novel oncological treatments, such as radio- and chemotherapy. However, these treatments impair the reproductive ability of patients, and may cause premature ovarian failure in females and azoospermia in males. Fertility preservation in both female and male oncological patients is nowadays possible and should be integrated as part of the oncological healthcare. The main objective of this review was to describe the different existing options of fertility preservation in patients undergoing gonadotoxic cancer treatments, as well as the differences in success rates that may appear in the different
\end{abstract}

Correspondence to: Professor Pepita Giménez-Bonafé, Department of Physiological Sciences, Physiology Unit, Faculty of Medicine and Health Sciences, Bellvitge Campus, Universitat de Barcelona, IDIBELL, L'Hospitalet del Llobregat, Carrer Feixa Llarga, s/n, 08907 Barcelona, Spain

E-mail:pgimenez@ub.edu

Abbreviations: AKT, protein kinase $\mathrm{B} ; \mathrm{As}_{2} \mathrm{O}_{3}$, arsenic trioxide; ASCO, American Society of Clinical Oncology; AYAs, adolescent and young adults; COS, controlled ovarian stimulation; ESGO, European Society of Gynecological Oncology; FEPNC, Spanish Federation of Parents of Children with Cancer; FOXO3, Forkhead box $\mathrm{O} 3$; FP, fertility preservation; G-CSF, granulocyte colony-stimulating factor; GnRH, gonadotropin-releasing hormone; IVF, in vitro fertilization; IVM, in vitro maturation; ICSI, intracytoplasmic sperm injection; INCIP, International Network on Cancer, Infertility and Pregnancy; NB, nanobins; OTC, ovarian tissue cryopreservation; PCOS, polycystic ovarian syndrome; PGD, pre-implantation genetic diagnosis; PI3K, phosphatidylinositol 3-kinase; POF, premature ovarian failure; POI, primary ovarian insufficiency; PTEN, phosphatase and tensin homolog

Key words: fertility preservation, female, male, prepuberal, gonadotoxic treatment, oncological treatment, oncofertility techniques evaluated. Emerging techniques are promising, such as the cryopreservation in orthotopic models of ovarian or testicle tissues, artificial ovaries, or in vitro culture prior to the autotransplantation of cryopreserved tissues. However, oocyte vitrification for female patients and sperm banking for male patients are considered the first line fertility preservation option at the present time for cancer patients undergoing treatment. Certainly, new fertility preservation techniques will continue to develop in the following years. However, despite the growing advances in the subject, optimal counselling from healthcare professionals should always be present.

\section{Contents}

1. Introduction

2. Fertility preservation techniques in females and males

3. Fertility preservation techniques in females

4. Emerging techniques

5. Fertility preservation techniques in males

6. Conclusions and future perspectives

\section{Introduction}

More than 70,000 adolescent and young adult (AYA) patients, with ages between 15 and 39 years, are diagnosed with cancer every year only in the United States. The incidence of cancer in children younger than 15 years of age is also approximately 10,000 cases per year (1).

In Europe, $>15,000$ AYAs are diagnosed with cancer each year, according to the International Society of Paediatric Oncology (https://siop-online.org/). In 2004, the Spanish Registry of Childhood Tumors (RETI-SEHOP) concluded that approximately 1,100 children in Spain are diagnosed with cancer annually (https://www.uv.es/rnti/index.html). The incidence of cancer in children in Spain is similar to that in other European countries, with approximately 160 new cases per million children each year, aged between zero and 
14 years, according to the Spanish Federation of Parents of Children with Cancer (FEPNC; http://cancerinfantil. org/cancer-infantil-cifras/). The survival rates of these patients have been steadily increasing in recent years due to the greater efficacy of novel oncological treatments. In fact, the 5-year survival rate for pediatric cancer patients approaches $80 \%$ (2). However, treatments entailing chemotherapy or radiotherapy, often result in impaired or absent reproductive ability. Moreover, only approximately half of patients report any reproductive issues, having previously been informed about the therapeutic options of fertility preservation (FP), which has been identified as the second most important aspect among young patients, following survival. In order to improve the quality of life and the survival of patients, the improvement of FP techniques has become an important topic in the field of research over the past years (3). In fact, the concept of oncofertility has recently appeared in the form of an interdisciplinary integrated network, based on medical methods, which is designed to maximize the reproductive future of oncological patients by offering various FP techniques. Furthermore, a standardization of the FP healthcare for oncological patients is required. Currently, two major international networks have been created for this purpose: The International Network on Cancer, Infertility and Pregnancy (INCIP) and the European Society of Gynecological Oncology (ESGO) (2).

\section{Fertility preservation techniques in females and males}

A literature review was conducted by searching for publications in PubMed between September, 2017 and May, 2018 with the following keywords and their combinations: 'fertility', 'preservation', 'women', 'male', 'prepuberal', 'young adults', 'gonadotoxic treatment', 'cancer treatment' and 'oncofertility'.

At the 20th week of embryogenesis, women reach the maximal number of germ cells in the genital rides, with approximately 6-7 million potential oocytes, known as the primordial follicles. However, only 1 million of these will remain at birth and only approximately 400,000 oocytes will survive to puberty. This number, known as the ovarian reserve, will decrease, reaching 1,000 oocytes at the time of menopause, due to the approximately 450 monthly ovulatory cycles, during which process most oocytes undergo atresia (degeneration and reabsorption) (4). The preservation of the ovarian reserve is necessary to maintain overall women's health, as it plays a role not only in oocyte development and fertility, but also in other systems, such as the cardiovascular system and osseous system (5).

The degree of the depletion of the ovarian reserve differs between chemotherapy and radiotherapy. As regards chemotherapy, it varies depending on the age of the patient (the younger the patient, the lesser the risk of ovarian failure), the chemotherapy agent used (alkylating agents being of greatest risk) and the duration of the treatment. Oocytes are very sensitive to radiation. Exposure to 20-30 Gy of radiation or total body radiation of $15 \mathrm{~Gy}$ lead to the loss of ovarian function [premature ovarian failure (POF)] (3).

The following techniques are available to women who are willing to preserve their fertility prior to, or during chemoand radiotherapy: Embryo cryopreservation, immature or mature oocyte cryopreservation, ovarian tissue cryopreservation (OTC) and ovarian transposition. In this review, we specifically focus on the oocyte and OTC. Other experimental techniques, such as the activation of ovarian follicles, in vitro follicle culture, artificial ovaries and novel fertoprotective agents, may appear to be promising, although further research is still required (6).

In males, the onset of production of spermatozoa begins at puberty and it is known as spermarche. Unlike women, from the moment of the spermarche, spermatogenesis is maintained during the entire duration of a man's life, on account of the spermatogonia type A, among others (7). Testicular stem cells differentiate into spermatogonia, which will eventually become spermatozoa under the process of spermatogenesis. Spermatogonia in the testes are extremely sensitive to radiation, regardless of age. Leydig cells, on the other hand, are more sensitive to radiation prior to the onset of puberty, whereas in adulthood, they become more resistant to radiation (8). Consequently, adult patients may preserve Leydig cell function and testosterone production following radiotherapy despite being azoospermic. Furthermore, if a population of spermatogonial stem cells (SSCs) remains after cancer treatment, as the effect is dose-dependent, the regeneration of spermatozoa may continue for years (9). Those at the highest risk of developing permanent sterility are children and adolescents with testicular cancer, leukemia and Ewing's sarcoma. Sperm banking is the recommended FP technique for males, although the cryopreservation of SSCs is also available.

\section{Fertility preservation techniques in females}

Embryo cryopreservation. This technique has established success rates and is a widely used and reliable method. It is like an in vitro fertilization (IVF) protocol, which has been performed for over 30 years. Women undergo controlled ovarian stimulation (COS) with gonadotropin injections to promote multifollicular growth. After 10-14 days, oocyte retrieval is performed, normally under conscious sedation and with transvaginal ultrasound-guided needle aspiration (10). The oocytes are then fertilized in the laboratory and are cryopreserved for future use, commonly in their blastocyst phase (4).

The disadvantages of this technique are mainly three as follows: The need of a stable male partner, ethical issues regarding embryo disposition and the time required for ovarian stimulation. COS normally begins during the early follicular phase. When a patient is diagnosed in her early follicular phase, ovarian stimulation with gonadotropin-releasing hormone $(\mathrm{GnRH})$ antagonist begins immediately. However, if the patient is in any other phase, the IVF standard protocols require the patient to wait up to 3 weeks before the process begins (10). Therefore, this method is not a viable option for women whose aggressive cancer treatment is of highest priority, as the IVF standard protocols require a wait of up to 3 weeks before the process begins (10). It is also not recommended in women with hormone-sensitive cancers and is not possible for prepubertal girls. It is also not recommended in women with hormone-sensitive cancers and not possible for prepubertal girls. 
There are three main cryopreservation techniques: Slow-freezing, ultra-rapid and vitrification. Slow-freezing involves a step-wise programmed decrease in temperature (11), achieving a freezing equilibrium due to the exchange of the extra- and intracellular fluids without causing meaningful osmotic and deformation cellular effects. However, ice crystals can be formed within the cells, which can result in extremely harmful effects (12). The procedure is long-lasting (approximately 1 or $2 \mathrm{~h}$ ) and requires expensive instrumentation and large quantities of liquid nitrogen, among others. Vitrification converts water into solid glass-like cells, avoiding ice crystal formation, both intracellular and extracellular (13). Expensive instrumentation it is not required, and only several minutes are needed. Furthermore, a meta-analysis in 2013 revealed that the rates of oocyte survival, fertilization and implantation where higher in vitrification than in slow-freezing methods (14). For these reasons, vitrification is nowadays the preferred technique.

Data on pregnancy and live both rates in cancer patients after frozen embryo transfer are limited. Live birth rates in non-oncological patients $<35$ years of age amount to $38.7 \%$ per frozen embryo transfer and to $34,8 \%$ for oocyte donor cycles (15).

Oocyte cryopreservation. As an alternative to embryo cryopreservation, this technique is the preferred option for postpuberal and adolescent females, women without a stable partner, and for those who do not wish to use a sperm donor. It overcomes the ethical and religious issues that emerge from the embryo preservation. Clinical outcomes in the oocyte vitrification strategy are superior to slow-freezing and thawing (16). With oocyte vitrification, women are able to conceive in the future and maintain their reproductive autonomy. However, it is not appropriate for patients who are in urgent need of treatment or patients with hormone-sensitive cancers, as the procedure also includes COS. The oocytes can be cryopreserved as mature eggs or as immature germinal vesicle oocytes. Mature oocyte cryopreservation is performed with the oocytes whose development is terminated in metaphase II. Nowadays, this is the preferred method for postpuberal patients and for patients whose chemotherapy and radiotherapy can be delayed. Immature oocytes obtained by aspiration and followed by in vitro maturation (IVM) techniques is a suitable option for prepubertal girls and women with hormone-sensitive cancers or with polycystic ovarian syndrome (PCOS), since COS is not required. This also allows the possibility of immediate cancer treatment. Oocytes will be matured in vitro (through IVM) as the cryopreservation of mature oocytes has yielded better survival outcomes than immature cryopreserved oocytes (17). The retrieval of immature oocytes can also be achieved during an OTC procedure.

OTC. Although this technique is still considered experimental, it is currently the only option for pediatric patients and for patients with hormone-dependent diseases, as it is COS-independent and does not delay the oncological treatment. It does not require a male partner or a sperm donor.

OTC is an invasive procedure, as it requires general anesthesia to surgically remove the ovarian tissue. This tissue, with a high content on follicles, is cryopreserved and can then be used as for the following procedures: i) Orthotopic implantation (reimplantation into the pelvic cavity; e.g., remaining ovarian tissue or peritoneum) or heterotopic implantation outside of the ovaries (e.g., rectum, pectoralis muscle, abdominal wall and chest wall); ii) isolation of follicles from the thawed tissue for in vitro growth, maturation and fertilization. During OTC, it is possible to aspirate immature oocytes from antral follicles of the ovarian tissue. Isolated oocytes can be cryopreserved or matured in vitro (through IVM) for later vitrification (18).

Either ovarian cortical tissue cryopreservation (slow-freezing) or whole ovary cryopreservation can be performed. All egg-containing follicles are in the outer one-millimeter layer of the ovary, and thus the removal of this layer of tissue is sufficient for cryopreservation. The success rate of live-birth after reimplantation is approximately $30 \%$ (6). The cryopreservation of the whole ovary remains a technical challenge due to the bigger size of the tissue, which hinders a homogeneous and adequate dispersion of cryoprotectant, and the vascular damage in form of ice crystals. Further studies are required in order for this technique to be used in standard clinical practice.

Up to 2015, 60 live birth cases had been reported with OTC in adult patients. However, the total number of re-implantation performed in each center until that time was unknown; thus, no success rates could be concluded (19). Prior to menarche, only one live birth following the autografting of cryopreserved tissue has been published (20), at least to the best of our knowledge. In 2015, Donnez et al (19) published a large case series $(\mathrm{n}=111)$ which revealed a pregnancy rate proportion of $29 \%(\mathrm{n}=32)$. Two women delivered 3 babies each, proving the efficacy of the technique and the possibility of conceiving naturally after only one procedure.

The most worrisome concern of OTC is the possibility of the re-introduction of carcinogenic cells into the cured patient or the subsequent malignant transformation of the ovarian tissue, which has been already reported (21). For this reason, a thorough examination of the ovarian tissue prior to cryopreservation and reimplantation is required.

Ovarian transposition (oophoropexy). This procedure aims to prevent ovarian damage during radiation therapy by relocating the ovaries away from the radiation field. Therefore, it will be of use in women who will undergo pelvic or low abdominal radiation therapy without additional gonadotoxic chemotherapy (22). According to the radiation field outlined by the radiation oncologist, the surgeon will decide the optimal location in the abdominal wall for ovarian transposition. Altogether, the ovaries will not be harmed by the therapy and ovarian failure will be prevented. The procedure is normally performed laparoscopically before the commencement of radiation. Success rates are not conclusive, as they vary from 16 to $90 \%$ (23).

Fertoprotective adjuvant agents. Another approach to preserving fertility is to protect the follicles during oncological treatment by administrating fertoprotective agents. One example is the use of GnRHa agonists, which are administrated 10 days prior to the commencement 
of thechemotherapy. GnRH analogues interfere with the hypothalamic-pituitary-gonadal axis and inhibit the ovarian function by suppressing gonadotrophin levels to prepubertal levels (3). Two meta-analysis of randomized trials concluded a reduced risk of POF in young breast cancer patients $(24,25)$, whereas its use was unclear in ovarian cancer and lymphoma (25). Another study demonstrated no effect in young patients with lymphoma (26). The quality of the evidence is insufficient to draw meaningful conclusions; high-quality studies are required to examine the long-term effects of the use of GnRHa on premature ovarian insufficiency (POI).

\section{Emerging techniques}

Activation of ovarian follicles. Cryopreserved ovarian tissue from prepubertal patients and patients with POF contains immature primordial follicles, which need to be activated in order to begin developing. This can be induced either in vivo [by interrupting the Hippo signaling pathway (27)] or in vitro, prior to autotransplantation, by activating the phosphatidylinositol 3-kinase (PI3K)/phosphatase and tensin homolog (PTEN)/protein kinase B (AKT)/Forkhead box O3 (FOXO3) pathway, which regulates primordial follicle activation in oocytes (27). This pathway also plays a crucial role in the follicle-stimulating hormone (FSH) stimulation of granulosa cell differentiation in antral follicles and in oocyte maturation of preovulatory follicles (27). This may be a promising fertility option for prepubertal patients and patients with primary ovarian insufficiency, whose cryopreserved tissue contains immature primordial follicles suitable for this technique. In vitro protocols involving the PTEN/AKT pathway are being developed in order to increase the pool of viable activated follicles available for in vitro growth (IVG) procedures (28).

In vitro follicle culture. This technique may be an option for patients who require urgent oncological treatment, and therefore are not good candidates for oocyte or embryo cryopreservation, such as patients with acute leukemia or acute myeloblastic leukemia (AML). OTC is the available option momentarily for these patients. However, the possibility of re-seeding original cancer cells from the ovarian tissue exists, and therefore other alternatives need to be raised.

The ovarian follicle culture in vitro, aims to mitigate the risk of re-implanting malignant cells from the cryopreserved ovarian tissue. It is therefore useful in patients with cancers whose metastasis appear often in the ovary or patients with BRAC1 and BRAC2 mutations, due to the increased risk of an ovarian cancer, which would not make possible the transplantation of cryopreserved ovarian cortex (29). However, the complete maturation of primordial follicles has not been achieved in humans yet (30).

In this procedure, individual follicles are isolated from the patient's bank tissue, which will afterwards be matured in vitro to become a functioning oocyte. These will be fertilized, and the embryos will be transferred to the uterus. The follicles can be cultured in two-dimensional (2D) or three-dimensional (3D) systems. These 3D culture methods are the most successful in maintaining the sphericity and the communications between cells (29) and have also shown greater follicular viability, follicle and oocyte diameters and hormone production (3).

Artificial ovaries. The creation of an artificial ovary for transplantation is a very promising fertility-restoring technique. Isolated preantral follicles obtained from ovarian cryopreserved tissue, together with other ovarian cells in a 3D-matrix, or scaffold, result in a ovary-like environment, which could allow the growth of follicles and therefore could restore both fertility and endocrine function of the ovary once they are transplanted (3). Luyckx et al (31) achieved the survival and growth of murine ovarian follicles (primary, secondary and antral follicles) within 1 week following the transplantation of ovarian cells in a fibrin matrix. Moreover, Laronda et al (32) accomplished the initiation of puberty in ovariectomized mice following an artificial ovary transplant.

Specific target tissue drugs. Both nanoparticles and fertopotective agents share the aim of protecting ovarian cells during gonadotoxic oncological treatments. These are discussed below:

i) Nanoparticles. This procedure entails the encapsulation of the therapeutic agent in order to reduce its plasma clearance and therefore its toxicity. For such a purpose, a nanoparticulate formulation of the therapeutic agent is developed and encapsulated within liposomal vesicles or 'nanobins' (NB) (33). Ahn et al (34) demonstrated a superior antitumor efficacy of the nanoparticulate formulation of arsenic trioxide $\left(\mathrm{As}_{2} \mathrm{O}_{3}\right)$ in nanobins $[\mathrm{NB}(\mathrm{Ni}, \mathrm{As})]$ in a murine model of lymphoma as well as a reduced fertotoxicity.

ii) Novel fertoprotective agents. Current research focus on two different pathways: a) Anti-apoptotic agents, such as imatinib, sphingosine-1-phosphatase (AS101), granulocyte colony-stimulating factor (G-CSF), thyroid hormone (T3) and tamoxifen (28), and they have shown to diminish follicle loss in animal models (35); and on b) agents which prevent follicle activation, such as AS101, an immunomodulator interacting with the PI3K/PTEN/AKT follicle activation pathway (36) and the anti-Mullerian hormone (35). In summary, a number of novel fertoprotectives agents to protect oocytes against gonadotoxic treatments are being investigated and may be available soon (3).

\section{Fertility preservation techniques in males}

In males undergoing gonadotoxic treatment, both sperm cryopreservation or testicular tissue cryopreservation are currently available $(3,6)$. The American Society of Clinical Oncology (ASCO) guidelines recommend that oncologists inform about the risk of infertility in patients with cancer during their reproductive stages of life, as well as to refer them to specialists in fertility treatment.

Cryopreservation of spermatozoa. The cryopreservation of ejaculated semen is the recommended FP technique for adult males and pubertal boys producing sperm in the ejaculate, who will be undergoing gonadotoxic treatment (37). For 
patients receiving radiation therapy only, gonadal shielding may be an option if sperm collection is not possible.

The spermarche begins at puberty, but it is not exactly known when this onset begins, since clinical parameters, such as Tanner stage or increase on reproductive hormones, do not always correlate with spermermatogenesis onset, according to some data from urine examination and electro-ejaculation in pubertal boys $(38,39)$. The successful sperm collection following masturbation has been reported for boys aged 12 years and older $(40,41)$.

The procedure includes the collection of, ideally, at least 3 semen samples, with an abstinence period of at least $48 \mathrm{~h}$ in between samples, and the following cryopreservation of the sperm samples, although often more than one semen sample must be taken in the same day to avoid the oncological treatment delay (7). In the case of ejaculation failure or when no spermatozoa are found in the ejaculate, sperm can be retrieved by epidydimal sperm aspiration, either percutaneous (PESA) or with microsurgery (MESA), testicular sperm extraction (TESE) or electro-ejaculation $(42,43)$.

Assisted reproductive treatment such as IVF and intracytoplasmic sperm injection (ICSI) are afterwards required. ICSI has the advantage of also allowing reproduction when the semen is of very poor quality or with only a few spermatozoa (7).

The pregnancy rates vary from $12 \%$ for intrauterine insemination to $32 \%$ for ICSI. To date, no follow-up data for large cohorts of children born after assisted reproductive treatment using frozen-thawed sperm of men with cancer are available in the literature, at least to the best of our knowledge.

It is worth mentioning that the European Germ Cancer Consensus Group and ASCO strongly recommend informing patients about the possibility of cryopreservation techniques before undergoing orchiectomy or gonadotoxical treatment (30). Unfortunately, such recommendations are oftentimes not followed by health-care professionals, and many patients remain without counseling in the matter.

Cryopreservation of SSCs in prepubertal children. Prepubertal children do not undergo spermatogenesis yet, and therefore they do not have mature sperm in their testes. Hence, the cryopreservation of spermatozoa is not possible. The only possibility for them is to preserve testicular tissue, which contains SSCs.

In an analogous manner to the cryopreservation of ovarian tissue in women, the testicular tissue can be obtained (through a testicular biopsy) and cryopreserved in form of spermatogonia or in form of testicular tissue (using slow-freeze or ultra-rapid techniques). This will be thereafter available to use when the patient is free of oncological illness and desires to have children. Once the tissue is thawed, it would allow in vitro spermatogenesis (44) or autotrasplantation of the cryopreserved tissue, either by infusion of a cell suspension into the seminiferous tubules or intratesticular grafting of the tissue (7).

The reintroduction of testicular stem cells into the seminiferous tissue could restart the sperm production (7). Orthotopic transplantation entails the risk of re-seeding malignant cancer cells (e.g., in patients with leukemia), as occurs with ovarian autotransplantation. To mitigate the problem, a decontaminated cell suspension could be a possible solution (45). In vitro culture of testicular cells to obtain mature spermatozoa also circumvents the risk of reseeding malignant cells in the auto-transplant of testicular tissue, being another branch of research at the moment (46). It is important to stress that fertility restoration strategies by auto-transplantation of cryopreserved testicular tissue have not been tested yet for safe clinical use in humans and therefore it is still considered experimental (6). More research is still needed regarding the use of frozen-thawed tissue to obtain mature spermatozoa in vitro (9).

\section{Conclusions and future perspectives}

Oncological healthcare is nowadays far from being solely the cure of cancer. Providing hope of future fertility following oncological treatment, significantly increases the quality of life of the patients and helps them to cope emotionally with cancer (30). FP in both female and male oncological patients is nowadays possible and should be integrated as part of the oncological health care. Different techniques exist and the most appropriate should be chosen depending on the characteristics of the patients: Male, female, prepubertal or postpubertal. Some of these have already proven successful outcomes whereas others, newer and more innovative, are still in need of further improvement and development.

Sperm banking is now considered the first line FP option for male patients; oocytes vitrification is currently considered the first line option for postpuberal female patients in which it is possible to delay chemotherapy and hormonal stimulation is authorized. Embryo banking gets in ethical conflict when it comes to preserving fertility, as healthcare's aim is to solely preserve the woman's fertility, which is the reason why it is not considered the first-line treatment anymore. Furthermore, growing evidence of safety and efficiency success in oocyte vitrification, upholds this technique to be the preferred one. When facing a therapeutic emergency, or contraindication for hormonal stimulation exists, OTC or puncture of immature oocytes are available. Immature oocytes will then be cryopreserved, directly or after being matured in vitro, to be vitrified as mature oocytes or as embryos after a fertilization technique (Fig. 1).

Among all the patients to whom these techniques address, pediatric and adolescent patients are the ones with the most restricted FP options (Fig. 2), higher survival rates, and thus those with the longest life expectancy. Therefore, special effort should be made to improve quality of life in this unique population and fulfill their reproductive wish.

As oncofertility is a recent concept and it is rapidly gaining importance, novel procedures involving emerging technological advances are being developed. The in vitro activation of ovarian follicles has proven itself to be a very promising technique for future approaches, as it could be addressed to patients with restricted FP options: Prepubertal children, hormone-sensitive tumors and those at urge to start treatment. OTC and subsequent transplantation, although still considered experimental, is currently the only hope for prepubertal children (Fig. 2). This technique has shown encouraging results in adult patients, but literature regarding pregnancy in 


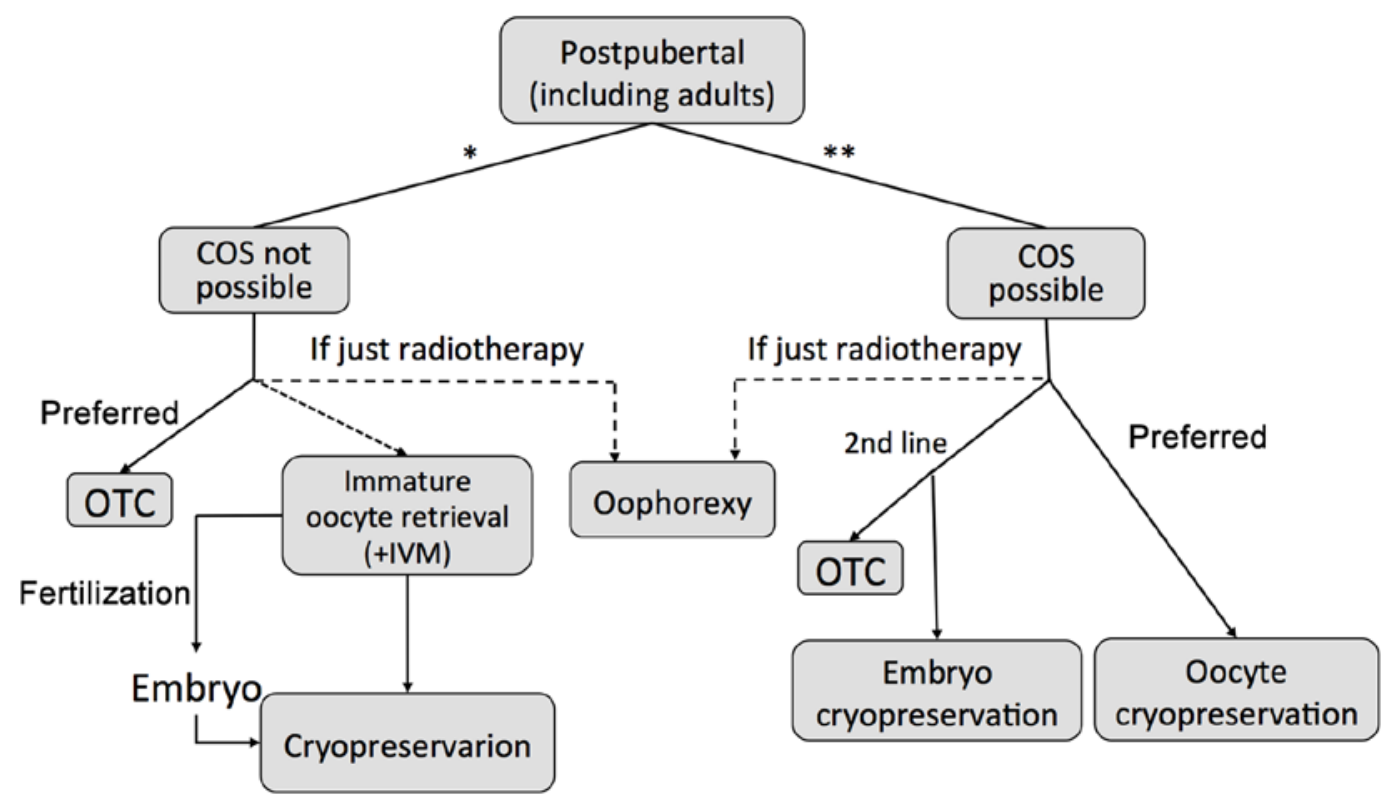

Figure 1. Fertility preservation techniques in postpuberal patients. The single asterisk symbol (") indicates that COS is not possible; the double asterisk symbol $\left(^{* *}\right)$ indicates that COS is possible (chemotherapy, radiotherapy or a combination of both, when the initiation of oncological treatment does not have to be immediate). COS, controlled ovarian stimulation; OTC, ovarian tissue cryopreservation; IVM, in vitro maturation.

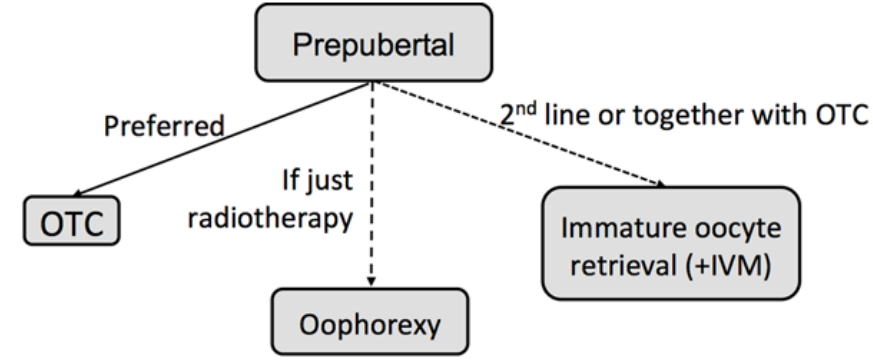

Figure 2. Fertility preservation techniques in prepubertal patients. OTC, ovarian tissue cryopreservation; IVM, in vitro maturation.

prepubertal children is very scarce. Only one live birth after autograft of cryopreserved tissue before menarche has been published (20).

Development of specific target chemotherapeutical treatment such as NB and the creation of artificial ovaries from stem cells, would respectively avoid and completely restore the ovarian function. Therefore, further development in these emerging techniques may lead to ground breaking advances of this field in the not so far future. Certainly, new FP techniques will continue to develop in the following years. However, despite the growing advances in the subject, optimal counselling from healthcare professionals is lacking.

In conclusion, FP in both female and male oncological patients is nowadays possible and should be integrated as part of the oncological health care. Different techniques exist and the most appropriate should be chosen depending on the characteristics of the patients: male, female, prepubertal or postpubertal. Many of the techniques are still in under experimental trials, whereas some others are standardized and established. Oocyte vitrification for female patients and sperm banking for male patients are now considered the first line FP option.

\section{Acknowledgements}

The authors would like to thank Mr. Aris Protopapas (Ludwig Maximilian University of Munich, Munich, Germany) for his helpful comments that greatly improved upon the manuscript.

\section{Funding}

The present study was supported grants from the Instituto Carlos III (PI 11/01377; PI15/01763 and PI18/01484, and ISCIII-RETICRD12/0036/0029), Fundación Vencer el Cancer (VEC), and from the Government of Catalonia (2017SGR-1014).

\section{Availability of data and materials}

Not applicable.

\section{Authors' contributions}

SDPL and PGB conceived and designed the study. SDPL collected the information (performed literature search) and wrote the manuscript. CS, FMS, AT and PGB assisted with the collection and selection of the information (literature search). FMS, AT and MP contributed to the conception and design of the study and wrote different sections, reviewed the manuscript and suggested some changes. MP and PGB edited the manuscript up to the final form. All authors have read and approved the final manuscript.

\section{Ethics approval and consent to participate}

Not applicable.

\section{Patient consent for publication}

Not applicable. 


\section{Competing interests}

The authors declare that they have no competing interests.

\section{References}

1. Coccia PF, Pappo AS, Altman J, Bhatia S, Borinstein SC, Flynn J, Frazier AL, George S, Goldsby R, Hayashi R, et al: Adolescent and young adult oncology, version 2.2014. J Natl Compr Canc Netw 12: 20-32, 2014.

2. Melan K, Amant F, Veronique-Baudin J, Joachim C and Janky E: Fertility preservation healthcare circuit and networks in cancer patients worldwide: What are the issues? BMC Cancer 18: 192, 2018.

3. Kim SY, Kim SK, Lee JR and Woodruff TK: Toward precision medicine for preserving fertility in cancer patients: Existing and emerging fertility preservation options for women. J Gynecol Oncol 27: e22, 2016 .

4. Donnez J and Dolmans MM: Fertility preservation in women. Nat Rev Endocrinol 9: 735-749, 2013.

5. Marder W, Fisseha S, Ganser MA and Somers EC: Ovarian damage during chemotherapy in autoimmune diseases: Broad health implications beyond fertility. Clin Med Insights Reprod Heal 2012: 9-18, 2012.

6. Martinez F; International Society for Fertility Preservation-ESHRE-ASRM Expert Working Group: Update on fertility preservation from the Barcelona International Society for Fertility Preservation-ESHRE-ASRM 2015 expert meeting: Indications, results and future perspectives. Fertil Steril 108: 407-415.e11, 2017.

7. Tournaye H, Dohle GR and Barratt CLR: Fertility preservation in men with cancer. Lancet 384: 1295-1301, 2014.

8. Shalet SM, Tsatsoulis A, Whitehead E and Read G: Vulnerability of the human Leydig cell to radiation damage is dependent upon age. J Endocrinol 120: 161-165, 1989.

9. Rodriguez-Wallberg K and Oktay K: Fertility preservation during cancer treatment: Clinical guidelines. Cancer Manag Res 6: 105-117, 2014.

10. Cakmak H and Rosen MP: Ovarian stimulation in cancer patients. Fertil Steril 99: 1476-1484, 2013.

11. Abdel Hafez FF, Desai N, Abou-Setta AM, Falcone T and Goldfarb J: Slow freezing, vitrification and ultra-rapid freezing of human embryos: A systematic review and meta-analysis. Reprod Biomed Online 20: 209-222, 2010.

12. Rezazadeh Valojerdi M, Eftekhari-Yazdi P, Karimian L, Hassani F and Movaghar B: Vitrification versus slow freezing gives excellent survival, post warming embryo morphology and pregnancy outcomes for human cleaved embryos. J Assist Reprod Genet 26: 347-354, 2009.

13. Vajta $G$ and Kuwayama M: Improving cryopreservation systems Theriogenology 65: 236-244, 2006.

14. Cil AP, Bang H and Oktay K: Age-specific probability of live birth with oocyte cryopreservation: An individual patient data meta-analysis. Fertil Steril 100: 492-9.e3, 2013.

15. Mahajan N: Fertility preservation in female cancer patients: An overview. J Hum Reprod Sci 8: 3-13, 2015.

16. Donnez J and Dolmans M-M: Fertility Preservation in Women. N Engl J Med 377: 1657-1665, 2017.

17. Lim JH and Chian RC: In vitro maturation of human immature oocytes. J Reprod Stem Cell Biotechnol: doi. org/10.1177/205891581000100205.

18. Meirow D, Levron J, Eldar-Geva T, Hardan I, Fridman E, Zalel Y, Schiff E and Dor J: Pregnancy after transplantation of cryopreserved ovarian tissue in a patient with ovarian failure after chemotherapy. N Engl J Med 353: 318-321, 2005.

19. Donnez J, Dolmans MM, Diaz C and Pellicer A: Ovarian cortex transplantation: Time to move on from experimental studies to open clinical application. Fertil Steril 104: 1097-1098, 2015.

20. Demeestere I, Simon P, Dedeken L, Moffa F, Tsépélidis S, Brachet C, Delbaere A, Devreker F and Ferster A: Live birth after autograft of ovarian tissue cryopreserved during childhood. Hum Reprod 30: 2107-2109, 2015.

21. Ernst EH, Offersen BV, Andersen CY and Ernst E: Legal termination of a pregnancy resulting from transplanted cryopreserved ovarian tissue due to cancer recurrence. J Assist Reprod Genet 30: 975-978, 2013
22. Tulandi T: Ovarian transposition before pelvic radiation UpToDate. 2018. https://www.uptodate.com/contents/ovariantransposition-before-pelvic-radiation.

23. Sonmezer M and Oktay K: Fertility preservation in female patients. Hum Reprod Update 10: 251-266, 2004.

24. Lambertini M, Ceppi M, Poggio F, Peccatori FA, Azim HA Jr, Ugolini D, Pronzato P, Loibl S, Moore HC, Partridge AH, et al: Ovarian suppression using luteinizing hormone-releasing hormone agonists during chemotherapy to preserve ovarian function and fertility of breast cancer patients: A meta-analysis of randomized studies. Ann Oncol 26: 2408-2419, 2015.

25. Del Mastro L, Ceppi M, Poggio F, Bighin C, Peccatori F, Demeestere I, Levaggi A, Giraudi S, Lambertini M, D'Alonzo A, et al: Gonadotropin-releasing hormone analogues for the prevention of chemotherapy-induced premature ovarian failure in cancer women: Systematic review and meta-analysis of randomized trials. Cancer Treat Rev 40: 675-683, 2014.

26. Demeestere I, Brice P, Peccatori FA, Kentos A, Dupuis J, Zachee P, Casasnovas O, Van Den Neste E, Dechene J, De Maertelaer V, et al: No Evidence for the Benefit of Gonadotropin-Releasing Hormone Agonist in Preserving Ovarian Function and Fertility in Lymphoma Survivors Treated With Chemotherapy: Final Long-Term Report of a Prospective Randomized Trial. J Clin Oncol 34: 2568-2574, 2016.

27. Hsueh AJW, Kawamura K, Cheng Y and Fauser BCJM: Intraovarian control of early folliculogenesis. Endocr Rev 36: $1-24,2015$.

28. Novella-Maestre E,Herraiz S, Rodríguez-Iglesias B,Díaz-García C and Pellicer A: Short-term PTEN inhibition improves in vitro activation of primordial follicles, preserves follicular viability, and restores AMH levels in cryopreserved ovarian tissue from cancer patients. PLoS One 10: e0127786, 2015.

29. Colgan TJ, Murphy J, Cole DE, Narod S and Rosen B: Occult carcinoma in prophylactic oophorectomy specimens: Prevalence and association with BRCA germline mutation status. Am J Surg Pathol 25: 1283-1289, 2001.

30. Ben-Aharon I, Abir R, Perl G, Stein J, Gilad G, Toledano H, Elitzur S, Avrahami G, Ben-Haroush A, Oron G, et al: Optimizing the process of fertility preservation in pediatric female cancer patients - a multidisciplinary program. BMC Cancer 16: 620, 2016

31. Luyckx V, Dolmans M-M, Vanacker J, Legat C, Fortuño Moya C, Donnez J and Amorim CA: A new step toward the artificial ovary: Survival and proliferation of isolated murine follicles after autologous transplantation in a fibrin scaffold. Fertil Steril 101: 1149-1156, 2014.

32. Laronda MM, Jakus AE, Whelan KA, Wertheim JA, Shah RN and Woodruff TK: Initiation of puberty in mice following decellularized ovary transplant. Biomaterials Feb 14, 2015 (Epub ahead of print).

33. Ahn RW, Chen F, Chen H, Stern ST, Clogston JD, Patri AK, Raja MR, Swindell EP, Parimi V, Cryns VL, et al: A novel nanoparticulate formulation of arsenic trioxide with enhanced therapeutic efficacy in a murine model of breast cancer. Clin Cancer Res 16: 3607-3617, 2010.

34. Ahn RW, Barrett SL, Raja MR, Jozefik JK, Spaho L, Chen H, Bally MB, Mazar AP, Avram MJ, Winter JN, et al: Nano-encapsulation of arsenic trioxide enhances efficacy against murine lymphoma model while minimizing its impact on ovarian reserve in vitro and in vivo. PLoS One: Mar 20, 2013 (Epub ahead of print)

35. Roness H, Kashi O and Meirow D: Prevention of chemotherapy-induced ovarian damage. Fertil Steril 105: 20-29, 2016.

36. Kalich-Philosoph L, Roness H, Carmely A, Fishel-Bartal M, Ligumsky H, Paglin S, et al: Cyclophosphamide Triggers Follicle Activation and "Burnout"; AS101 Prevents Follicle Loss and Preserves Fertility. Sci Transl Med: May 15, 185ra62, 2013.

37. Pacey AA and Eiser C: Banking sperm is only the first of many decisions for men: What healthcare professionals and men need to know. Hum Fertil (Camb) 14: 208-217, 2011.

38. Pedersen JL, Nysom K, Jørgensen M, Nielsen CT, Müller J, Keiding N and Skakkebaek NE: Spermaturia and puberty. Arch Dis Child 69: 384-387, 1993.

39. van der Kaaij MAE, van Echten-Arends J, Simons AHM and Kluin-Nelemans HC: Fertility preservation after chemotherapy for Hodgkin lymphoma. Hematol Oncol 28: 168-179, 2010.

40. Hagenäs I, Jørgensen N, Rechnitzer C, Sommer P, Holm M, Schmiegelow K, Daugaard G, Jacobsen N and Juul A: Clinical and biochemical correlates of successful semen collection for cryopreservation from 12-18-year-old patients: A single-center study of 86 adolescents. Hum Reprod 25: 2031-2038, 2010. 
41. Bahadur G, Ling KLE, Hart R, Ralph D, Wafa R, Ashraf A, Jaman N, Mahmud S and Oyede AW: Semen quality and cryopreservation in adolescent cancer patients. Hum Reprod 17: 3157-3161, 2002.

42. Chan PT, Palermo GD, Veeck LL, Rosenwaks Z and Schlegel PN: Testicular sperm extraction combined with intracytoplasmic sperm injection in the treatment of men with persistent azoospermia postchemotherapy. Cancer 92: 1632-1637, 2001

43. Köhn FM, Schroeder-Printzen I, Weidner W, Montag M, van der Ven $\mathrm{H}$ and Schill WB: Testicular sperm extraction in a patient with metachronous bilateral testicular cancer. Hum Reprod 16: 2343-2346, 2001.

44. Ning L, Meng J, Goossens E, Lahoutte T, Marichal M and Tournaye H: In search of an efficient injection technique for future clinical application of spermatogonial stem cell transplantation: Infusion of contrast dyes in isolated cadaveric human testes. Fertil Steril 98: 1443-8.e1, 2012.
45. Goossens E, Van Saen D and Tournaye H: Spermatogonial stem cell preservation and transplantation: From research to clinic. Hum Reprod 28: 897-907, 2013.

46. Stukenborg J-B, Schlatt S, Simoni M, Yeung C-H, Elhija MA, Luetjens CM, Huleihel M and Wistuba J: New horizons for in vitro spermatogenesis? An update on novel three-dimensional culture systems as tools for meiotic and post-meiotic differentiation of testicular germ cells. Mol Hum Reprod 15: 521-529, 2009. 